thought to have paved the way for the latter, but newer evidence is emerging to suggest it was the other way around.

Human ancestors might first have come together to mark the change of seasons observable in the movement of the Sun or other celestial bodies across the sky or along the horizon. E Groups (Fig. 1) contain a low mound or pyramid on the western side of an architectural complex with an elongated platform on the eastern side. Looking from the western structure aids the viewer to witness sunrise during the winter and summer solstices, which are visible along the northern and southern corners, respectively, of the eastern platform (which is elongated from north to south). Brilliantly simple in design, this type of construction was built, over and over again, up and down the Usumacinta region and throughout the Maya lowlands to the east.

Using the revealing 'eyes' of lidar, Inomata and colleagues document 16 instances of E-Group constructions during the first millennium BC. These were built on top of massive rectangular platforms. The platform at Aguada Fénix is the largest of any such platform discovered from this early time period, and Inomata and colleagues suggest that it might be the largest Maya construction built before Spanish invaders arrived. On the basis of the site's absence of excavated stone sculpture depicting rulers - such as the colossal heads found from the same time period in the Olmec region - the authors argue that these constructions were truly public architecture and not built at the behest of rulers. If so, then why were they built so large, and abandoned only hundreds of years later (as indicated by radiocarbon-dating information from the authors' excavations)? And how far to the east and west of Aguada Fénix can such arrangements of a huge platform with an $\mathrm{E}$ Group be found? Strictly speaking, this architectural pattern is not a strong characteristic of the central Maya lowlands to the east nor of the Olmec region to the west.

Many questions remain for further research, but there is no doubt that lidar is continuing to transform archaeological research in forested regions. At Aguada Fénix, in particular, the lidar data coupled with Inomata and colleagues' excavations substantially deepen our understanding of the social transformations that occurred there, and strengthen the argument that public architecture on a monumental scale pre-dated village life in eastern Mesoamerica. These findings will lead some to cast a critical eye on the proposed link between public architecture and hierarchical rulership, given that the latter seems to have commenced in the Maya lowlands hundreds of years after the construction of the Aguada Fénix site. The fact that Inomata and colleagues' research took three years, rather than three decades, also demonstrates the powerful way in which lidar is facilitating the rapid detection and investigation of the past by offering a way of peering through the veils of the forest canopy.

Patricia A. McAnany is in the Department of Anthropology, University of North Carolina, Chapel Hill, North Carolina 27599, USA.

e-mail: mcanany@email.unc.edu

\footnotetext{
1. Chase, A. F., Chase, D. Z., Fisher, C. T., Leisz, S. J. \& Weishampel, J. F. Proc. Natl Acad. Sci. USA 109, 12916-12921 (2012).
}

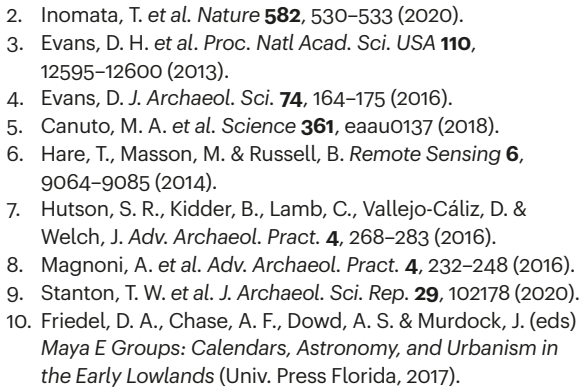

This article was published online on 3 June 2020.

\title{
Cancer
}

\section{Tumour metabolites hinder DNA repair}

\section{Lei-Lei Chen \& Yue Xiong}

\begin{abstract}
Altered metabolism and genome instability are hallmarks of cancer. A mechanism now explains how three small molecules that accumulate in tumours connect abnormal metabolism to genomic problems by hindering DNA repair. See $\mathbf{p . 5 8 6}$
\end{abstract}

Towards the end of the nineteenth century, chromosomal abnormalities detected under the light microscope revealed that a type of massive genome instability resulting in an abnormal number of chromosomes occurs in certain types of cancer. Not long after, the biochemist Otto Warburg observed that tumour cells tend to use pathways of glucose and energy metabolism that are distinct from those used by normal cells. We now know that genome instability and altered metabolism are two common characteristics of most tumour cells. Genome instability has been investigated continuously since its discovery; altered metabolism was rediscovered as a research area only recently. But not much crosstalk between these two processes in cancer has been reported so far. Sulkowski et al. ${ }^{1}$ reveal on page 586 how several metabolites that accumulate to high levels in tumour cells suppress DNA repair, thus revealing a direct link between altered metabolism and genome instability caused by DNA damage.

Mutations targeting the genes encoding the enzymes isocitrate dehydrogenase 1 and 2 (IDH1 and IDH2) result in cells accumulating high levels of the metabolite 2-hydroxyglutarate (2-HG). Mutations in the genes encoding the enzymes fumarate hydratase and succinate dehydrogenase cause cells to accumulate high levels of the molecules fumarate and succinate, respectively. These three small molecules are often referred to as oncometabolites because their accumulation boosts tumour development ${ }^{2,3}$, and they are structurally similar to the molecule $\alpha$-ketoglutarate $(\alpha-K G)$. This is an intermediate in the Krebs-cycle pathway that also serves as a component, called a co-substrate, needed for the function of a family of enzymes called $\alpha-\mathrm{KG} / \mathrm{Fe}(\mathrm{II})-$ dependent dioxygenases.

This enzyme family, which comprises 65 members in humans ${ }^{4}$, catalyses a diverse range of oxidation reactions in proteins, DNA, RNA and lipids. In these reactions, $\alpha-K G$ binds to the active site of the enzyme to aid catalysis. However, 2-HG, succinate and fumarate can compete with $\alpha$-KG for binding to this catalytic site and thus inhibit these enzymes. One such enzyme is lysine histone demethylase (KDM), which modifies chromatin - the complex of DNA and proteins of which chromosomes are made $^{5-7}$.

Two closely related KDMs, called KDM4A and KDM4B, catalyse the removal of a methyl group (demethylation) from a lysine amino-acid residue (termed K9) in the DNA-binding histone 3 (H3) proteins in chromatin. The methylation of H3K9 is linked to a pathway called the homology-dependent repair (HDR) pathway, which mends double-strand breaks (DSBs) in DNA ${ }^{8}$. DSBs are the most dangerous type of DNA damage. If left unrepaired, they can cause chromosome breakage and genomic instability that might promote tumour growth or lead to cell death.

Sulkowski and colleagues investigated HDR in human cancer cells grown in vitro. They found that, at a DSB site, the local addition of three methyl groups to $\mathrm{H} 3 \mathrm{~K} 9$ to generate 
trimethylated $\mathrm{H} 3 \mathrm{~K} 9 \mathrm{me} 3$ residues has a key role in the initiation of HDR. In tumour cells that have mutations in the genes encoding IDH1, IDH2, fumarate hydratase or succinate dehydrogenase, the authors report that high levels of oncometabolites inhibit KDM4B. This inhibition of demethylation results in a widespread hypermethylation of $\mathrm{H} 3 \mathrm{~K} 9$ that masks the specific local appearance of H3K9me3 marks and impairs the recruitment of factors needed for HDR and DSB repair (Fig. 1).

A link between oncometabolites and DNA-repair defects was previously suggested by the clinical finding that people who have a type of cancer called glioma with mutations in the $I D H 1$ or $I D H 2$ genes benefited from a combination of chemotherapy and radiation therapy, both of which induce DNA damage9. That finding indicates that tumours that accumulate high levels of oncometabolites are vulnerable to therapy that causes DNA damage. Moreover, a genomic analysis of different types of cancer ranked $I D H 1$ as being the fifth most frequently mutated human gene that is connected to DNA repair ${ }^{10}$.

Two mechanisms have previously been proposed to explain how the $2-\mathrm{HG}$ that accumulates when IDH1 or IDH2 are mutated causes DNA-repair defects. One idea is that 2-HG directly inhibits the enzymes ALKBH2 and ALKBH3, which repair methylation-induced single-strand DNA damage ${ }^{11}$. Another suggestion is that 2-HG inhibits $\mathrm{H} 3 \mathrm{~K} 9$ demethylases and thereby causes a reduction in the expression of ATM, a key protein required for DNA repair ${ }^{12}$.

Sulkowski and colleagues had previously found that oncometabolites suppressed the HDR pathway and had identified KDM4A and KDM4B as being important for DSB repair ${ }^{11}$. The authors therefore explored possible connections between these processes. HDR is a complex event that involves the sequential recruitment of multiple repair factors to DSB sites, with the protein Tip60 being among the first to arrive at the damaged region ${ }^{8}$. Sulkowski et al. used a system in which human cells grown in vitro were engineered to allow the precise initiation of DSB and monitoring of the repair process.

The authors found that in control cells that did not have high levels of oncometabolites, a rapid spike of $\mathrm{H} 3 \mathrm{~K} 9 \mathrm{me} 3$ modifications occurred locally in chromatin in the vicinity of the DSB within 30 minutes of the DSB being induced. This was followed by the coordinated recruitment of factors needed for HDR. However, in cancer cells with high levels of oncometabolites, H3K9me3 was elevated throughout the genome before DSB induction, and the subsequent recruitment of the factors needed for HDR was substantially impaired compared with that in the control cells. These defects in repair-factor recruitment could be prevented by deleting the mutant version of
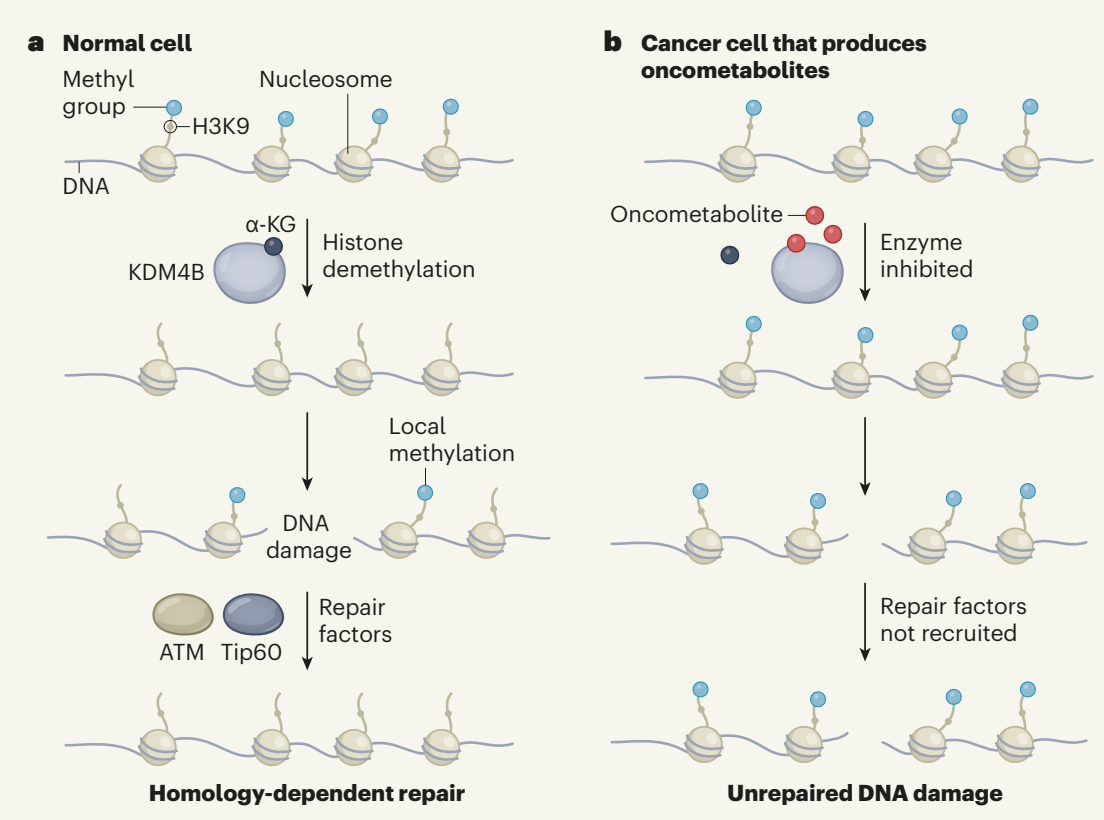

Figure 1 | How molecules in cancer cells inhibit the repair of DNA damage. a, DNA wraps around histone proteins to form a structure called a nucleosome. In normal cells, the enzyme KDM4B catalyses the removal of methyl groups from the lysine 9 (K9) amino-acid residue of the protein histone $3(\mathrm{H} 3)$ in the nucleosome. This H3K9 demethylation activity requires the small molecule $\alpha$-ketoglutarate ( $\alpha$-KG). If a double-strand break in DNA occurs, H3K9 is methylated at the damage site and this local methylation signal recruits DNA-repair factors that include the proteins Tip60 and ATM. These fix the damage through a process called homology-dependent repair. b, As a result of certain mutations, some cancer cells accumulate small molecules termed oncometabolites that promote tumour growth. Sulkowski et al. ${ }^{1}$ have revealed a mechanism that underlies this phenomenon. Oncometabolites compete with $\alpha-\mathrm{KG}$ for binding to KDM4B and thus inhibit the enzyme's function. This results in H3K9 methylation across the genome. This global hypermethylation masks a local spike in $\mathrm{H} 3 \mathrm{~K} 9$ methylation occurring after DNA damage, and hinders the recruitment of DNA-repair factors. Unrepaired DNA damage can lead to genome instability and thus boost tumour growth.

IDH1 or by treatment with a pharmacological inhibitor of mutant IDH1 protein to block 2-HG production. These results establish a causal relationship between the presence of oncometabolites and impaired DSB repair.

How might KDM4B inhibition by oncometabolites impair HDR? Local H3K9 methylation activates Tip60, which in turn activates ATM, a key enzyme needed for HDR. Results from a series of experiments support the authors' model that a sudden increase in H3K9me3 modifications at a DSB site serves as a key signal to recruit repair factors. Blocking the accumulation of oncometabolites, adding $\alpha-K G$, or engineering cells to express KDM4A or KDM4B (but not other KDMs or $\mathrm{ALKBH} 2$ or $\mathrm{ALKBH} 3$ ), resulted in a decrease in global genomic $\mathrm{H} 3 \mathrm{~K} 9 \mathrm{me} 3$ modifications and restored both the recruitment of repair factors and DSB repair at an engineered DNA-damage site, compared with the effects seen in cells that did not receive such treatment. If cells producing oncometabolites were engineered to have a mutant version of a histone that sequesters $\mathrm{H} 3 \mathrm{~K} 9$ methyltransferase enzymes and thus reduces the genomic level of H3K9me3 modifications, the cells displayed an $\mathrm{H} 3 \mathrm{~K} 9 \mathrm{me} 3$ spike on DSB formation that led to
Tip60 recruitment and repair of DNA damage. Sulkowski and colleagues' findings expand the known roles of oncometabolites and raise several interesting questions. How does the rapid spike in $\mathrm{H} 3 \mathrm{~K} 9 \mathrm{me} 3$ at a DSB site result in the coordinated recruitment of repair proteins, and what factor(s) might recognize such a modification of a DSB site? Around the DSB site, does hypermethylation of $\mathrm{H} 3 \mathrm{~K} 9$, which is known to recruit repressive factors that drive the formation of a condensed form of chromatin called heterochromatin, prevent the binding of factors needed for HDR? Questions also remain about whether the roles of KDM $4 \mathrm{~A}$ and KDM4B differ in HDR. Both enzymes catalyse the same type of $\mathrm{H} 3 \mathrm{~K} 9$ demethylation, and boosting their expression can overcome inhibition by oncometabolites and prevent HDR defects. Yet the authors report that the depletion only of KDM4B impairs HDR.

The enzyme PARP promotes the repair of single-strand DNA breaks, and inhibitors that block PARP are used to treat certain types of cancer. Tumour cells that produce 2-HG are particularly prone to death if treated with PARP inhibitors ${ }^{11}$. The findings by Sulkowski et al.might lead to new therapeutic strategies that exploit the therapeutic opportunities 
arising from oncometabolite accumulation, given that we now have a clearer picture of how such cancer cells are vulnerable if DNA-repair processes are targeted.

Lei-Lei Chen and Yue Xiong are in the Department of Biochemistry and Biophysics, and at the Lineberger Comprehensive Cancer Center, University of North Carolina at Chapel Hill, North Carolina 27516, USA. e-mail: yxiong@email.unc.edu

1. Sulkowski, P. L. et al. Nature 582, 586-591 (2020)

2. King, A., Selak, M. A. \& Gottlieb, E. Oncogene

\section{Quantum physics}

\section{A step closer to secure global communication}

\section{Eleni Diamanti}

Quantum key distribution is a cryptographic method that

can guarantee secure communication. A satellite-based experiment has shown that this technique can be applied over long distances without the need for trusted relays. See p.501

Modern society is driven by the large-scale exchange of information. As a result, secure communication of sensitive data around the world is an increasingly valuable asset. The mathematical toolbox that is widely used for this task can be complemented by applying the principles of quantum physics to enhance the security of the communication link. This approach has highly desirable features, such as protection of the encrypted information from threats that might arise as a consequence of future advances in computational power. However, it also comes with substantial technological challenges in terms of the range of communication possible and the degree of trust in the devices used. Yin et al. ${ }^{1}$ demonstrate on page 501 that such cryptographic solutions can be deployed over distances exceeding 1,000 kilometres, without compromising the security promised by the underlying quantum technology.

The flagship application of quantum communication is known as quantum key distribution (QKD). This process enables two parties located at a distance from each other to share a secret string of bits (units of information) called a key, which they can use to encrypt and decrypt secret messages, without making assumptions about the computational power of a potential eavesdropper. Although the principle of such absolute security is based solidly on fundamental laws of nature, practical implementations come in different configurations ${ }^{2}$. allowed to be untrusted.
25, 4675-4682 (2006).

3. Ye, D., Guan, K.-L. \& Xiong, Y. Trends Cancer 4, 151-165 (2018).

4. Rose, N. R., McDonough, M. A., King, O. N. F., Kawamura, A. \& Schofield, C. J. Chem. Soc. Rev. 40, 4364-4397 (2011).

5. Chowdhury, R. et al. EMBO Rep. 12, 463-469 (2011).

6. Xu, W. et al. Cancer Cell 19, 17-30 (2011)

7. Xiao, M. et al. Genes Dev. 26, 1326-1338 (2012).

8. Sun, Y. et al. Nature Cell Biol. 11, 1376-1382 (2009).

9. Cairncross, J. G. et al. J. Clin. Oncol. 32, 783-790 (2014).

10. Knijnenburg, T. A. et al. Cell Rep. 23, 239-254.e6 (2018).

11. Sulkowski, P. L. et al. Sci. Transl. Med. 9, eaal2463 (2017).

12. Inoue, S. et al. Cancer Cell 30, 337-348 (2016).
For example, it is possible for one of the two parties to prepare quantum states of light the natural physical carrier of information in quantum communication - and to send them to the second party, who measures them. By processing these data using standard classical communication, the two parties can then extract the secret key. QKD in this setting has been demonstrated over $400 \mathrm{~km}$ in a lowloss optical fibre ${ }^{3}$ and over $1,200 \mathrm{~km}$ using a satellite-to-ground communication link ${ }^{4}$.
Although impressive, these demonstrations require the two parties' devices to be fully characterized and trusted. Furthermore, losses in the optical-transmission medium eventually become prohibitive. As a result, the networks that need to be established to distribute keys securely between parties contain nodes, which also need to be trusted ${ }^{5,6}$. This constraint might be undesirable for some applications.

If, instead, one could use the distribution of 'entangled' states of light produced by a source, the need for trust would be greatly alleviated. Entangled states embody the peculiar nature of quantum physics and exhibit correlations not found in classical physics. Such correlations can be routed through devices called quantum repeaters, so that remote physical systems can become entangled. The past few years have seen major progress in this direction ${ }^{7}$. But, so far, the longest distances for entanglement distribution have been achieved by transmitting the states directly. These distances are approximately $100 \mathrm{~km}$ in an optical fibre $^{8,9}$ and 1,200 km using satellite links ${ }^{10}$.

Ideally for QKD, the security of the key generated would be confirmed just by detecting these non-classical correlations experimentally, through statistical properties known as Bell inequalities, without having to trust the devices used by the two parties ${ }^{11}$. However, in practice, achieving this level of security places stringent requirements on the experimental devices that cannot be satisfied by currently available technologies. A way forward is to implement entanglement-based QKD that has weaker requirements, whereby, although the parties' devices must be trusted, the source of the entangled states can remain untrusted $^{12}$.

Yin et al. have performed a complete, long-distance implementation of QKD with these restrictions (Fig. 1). A key way to

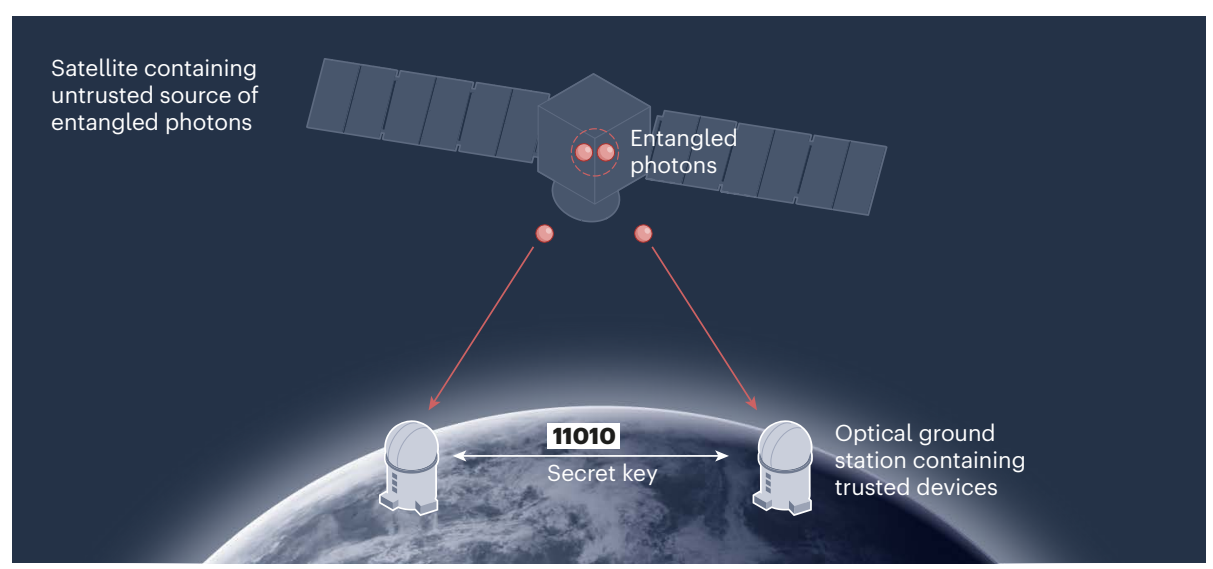

Figure 1 | Entanglement-based quantum cryptography. Yin et al. ${ }^{1}$ report an experiment in which pairs of entangled photons (photons that are correlated in a non-classical way) are produced on board the satellite Micius. The photons in each pair are then sent to two optical ground stations that are separated by a distance of 1,120 kilometres. This process enables parties at the two stations to share a secret string of bits called a key, which they can use to encrypt and decrypt secret messages with absolute security. In the authors' set-up, the devices used by the two parties must be trusted, but the source of the entangled photons is 